\title{
Telemedicine as an Aid to Clinical Practice, Research and Education in Plastic Surgery
}

\author{
Marek Dobke, M.D., Ph.D. and Elizabeth Whitehead, B.S. \\ University of California, San Diego \\ United States of America
}

\section{Introduction}

Telemedicine has the potential to expedite and improve the delivery of high-quality, costeffective care by extending the reach of health care practitioners (or patients themselves) beyond their local setting, using advanced information technologies. Plastic Surgery relies to a significant degree on visual modes of telemedicine to transfer medical information for diagnostic, physical examination, and outcome measure purposes; therefore, this specialty in particular, derives major benefits from telemedicine. At the same time, telemedicine benefits from Plastic Surgery by providing an opportune model for research and for testing new developments in this technology. The two basic modes of telemedicine applications: store and forward (asynchronous transfer) and real time transmission (synchronous transfer, e.g., videoconference) are both utilized in the plastic surgery setting. Intense reliance on both static and dynamic images distinguishes this specialty to an even greater degree than dermatology as a model for telemedicine research and development as well as for an educational tool (Meyer \& Friedman, 2010). Both physicians and patients have been surveyed for their perspectives on the introduction of telemedicine in plastic surgery, and their acceptance for the technology is high. Both parties assign telemedicine positive ratings in satisfaction surveys, although some potential problems with practical solutions have been noted and will be presented below.

Objectives of early studies included assessing the accuracy and usefulness of telemedicine consults in aesthetic surgery, reconstructive surgery, and problem wound care. Comparisons were made between consultations provided via telemedicine to those provided by a direct or face-to-face consultation. The specificity, sensitivity, and positive predictive values of equivalent (or different) diagnosis and potential treatment plan were calculated. We made the assumption that a correct diagnosis and indication (or lack thereof) for surgical intervention was established during direct consultation. Agreement levels between the two assessments (telemedicine and face-to-face) were judged by an independent surgeon. Typically, agreement rates were categorized as follows: total agreement, trivial disagreement (error not changing the overall management plan), and clinically important disagreement (error requiring change of approach after face-to-face consultation). Many studies show evidence of adequate overall accuracy of telemedicine as a tool in research, education and clinical practice in plastic surgery (Dobke MK et al., 2006; Dobke MK et al., 2007; Dobke MK et al., 2008; Dobke MK \& Gosman A, 2009; Gosman A et al, 2009). 


\title{
2. Positive impact of telemedicine on triage and management of problem wounds and other conditions by plastic surgeons, and as an example of practice pathway improvement
}

Telemedicine offers efficient access to specialists for patients with chronic problem wounds, eliminating the emotional and physical stress related to transporting patients to hospital/clinic settings. It allows for an immediate, relatively accurate, preliminary management step for patients prior to direct evaluation in the hospital, wound care clinic, etc. Dobke MK et al., 2006, studied the impact of telemedicine for triaging and developing management plans for patients with chronic, problematic wounds in long-term care, skilled nursing, and home care settings. Telemedicine was also assessed for its usefulness in enhancing communication with the surgical wound care specialist.

\author{
From: xxxxx@email.com \\ Sent: May 13, 2008 9:24 AM \\ To: xxxxx@email.com \\ Subject: Emailing: 050808 001.jpg
}

This patient is 96 years old, Wound measures $3.2 \times 2.5 \times 0.6$ red with thin scattered fibrin, with slightly bloody drainage. At present the treatment is Silvasorb. This patient is new to this homehealth, so no one knows much history on the wound. They say that this was something that happened many years ago and it heals then she scratches it and it reopens. I think maybe a biopsy should be done, What do you think?

The message is ready to be sent with the following file or link attachments:

050808 001.jpg

Note: To protect against computer viruses, e-mail programs may prevent sending or receiving certain types of file attachments. Check your e-mail security settings to determine how attachments are handled.

Fig. 1. Telemedicine for the Outreach Wound Program: A "field" nurse from a home health care facility sent this email to a surgical specialist detailing a summarized patient history with the corresponding photograph (right image) from a patient being originally referred to a Wound Care Clinic. Based on the patient history (17 year history of a non-healing apparently post-traumatic left malar area wound) through a telemedicine assessment, the patient - with the approval her primary care physician - was redirected to a Plastic Surgery Consultant since surgical intervention was likely. Patient management was streamlined based on telemedicine consultation: the problem wound was identified as a squamous cell carcinoma and she underwent appropriate ablative and reconstructive treatment. Experience of others confirms that referral by digital image for skin malignancy and other cutaneous lesions is a safe, accurate and cost effective referral pathway. This significantly reduces the time interval between referral diagnosis and onset of treatment for the malignant condition (Tadros A et al., 2009).

In this study a trained "field" nurse (RN and LVN) assessed 120 problem wound patients in an ambulatory care setting between January 2003 and December 2005. The nurse relayed a 
summarized history, laboratory results (if any), and photographs via email to the surgical specialist establishing an asynchronous transfer mode of telemedicine. Following an initial assessment and management plan, including diagnosis via telemedicine consult, the patient was also evaluated in person by a board certified plastic surgeon within two weeks of the initial consult request. The diagnosis and indication (or lack thereof) for surgical intervention was compared between telemedicine consult and face-to-face consult with the assumption that direct consult provided the correct treatment plan.

Telemedicine consults provided accurate assessment with only $1.67 \%$ (2/120 cases) change in diagnosis during the direct consultation (Dobke MK et al., 2006). The overall sensitivity of telemedicine-based problem wound assessments (as measured by correctness of establishing an indication for surgical intervention validated by the direct consult) was $94 \%$, specificity was $99 \%$, and the positive predictive value was $94 \%$. Overall, a telemedicine consult used as a decision aid is effective, relatively accurate, and streamlines the management process (Fig. 1).

The Association for the Advancement of Wound Care (AAWC) guidelines, advise treating chronic wounds with multidisciplinary teams (MDTs) of specialists due to the frequent complexity of pathology leading the wound and high incidence of co-morbidities (AAWC online, 2007). Additionally, problem wounds require prompt treatment so management systems should reduce or eliminate redundant time spent by MDTs developing treatment plans. However, in a retrospective study (Dobke MK et al., 2007), a database of 120 patients was analyzed to determine whether every patient management plan had to be formulated by the multidisciplinary team. Agreement levels between the initial management plan established by the surgeon after the telemedicine consult and the final management plan by the MDT based on direct, comprehensive consultation were judged by an independent investigator. There was a $93.6 \%$ concordance with the management plan established by the surgeon based on the telemedicine consult alone in comparison to the plan established by the MDT with direct patient contact. This study showed that telemedicine technology in the hands of an experienced physician could streamline management of a problem wound.

Regarding patient follow-up and measuring treatment outcomes, specialty facilities or home care wound management programs using telemedicine found that telemedicine facilitated communication between health caregivers from outside to inside the facilities. This improved outcomes and provided a new benefit for home care by eliminating the barrier to accessing distant plastic surgeons involved in wound care programs (or other specialists). This study established that the low availability of specialists in wound care programs and the corresponding hindrance for rapid treatment can be ameliorated by telemedicine. (Dobke MK et al, 2006; Terry M et al., 2009).

Similar to the application of telemedicine to the wound care population, the overall accuracy of telemedicine consults as applied to facial and breast aesthetic assessment demonstrated high sensitivity, specificity, and positive predictive value and greater than $95 \%$ confidence interval. This is close to "gold standard" care in terms of the accuracy of diagnostic tests comparing telemedicine to direct consultation. Furthermore, management proposals established on the basis of telemedicine consults were rarely changed after direct face-toface consultation. When changes were implemented they were typically dictated by patient preference (Dobke MK et al., 2009).

Limitations of telemedicine in plastic surgery are related to the quality of images and to the absence of data one would obtain by a direct face-to-face physical examination such as information obtained by the "tactile" part of physical examination (e.g., flap or replant tissue turgor). Optical problems may impact both static and dynamic visual observation 
(Fig. 2). Poor quality of images (300 dpi or less), "pixelization" of the image, and/or perspective distortion may lead to clinically important differences between an electronically transmitted photo and direct examination. This may result in an erroneous proposed diagnosis and management plan (Dobke MK et al, 2006).
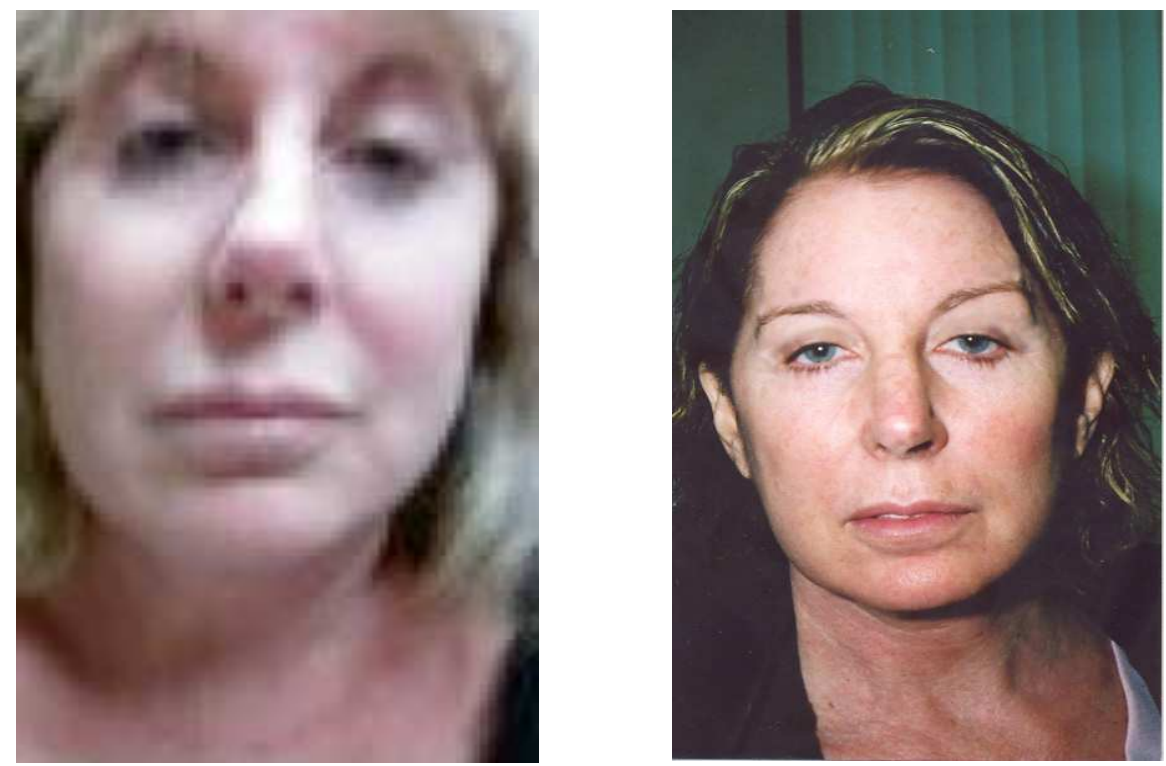

Fig. 2. Clinically important disagreement between the "telemedicine" and the "face-to-face" assessments. The image on the left shows a patient who transmitted her image from her cell phone and stated that she was kicked by a horse into the right side of her face and nose. With this history and the phone image strongly suggestive of the deviation of the nasal pyramid (left image) the tentative diagnosis of nasal fracture was established. On the following day the patient was seen in the clinic and her nose appeared to be only mildly swollen (right image). There were no signs of the nasal fracture or deformity. It appears that the cell phone photo held by the patient's right hand, diagonally, 45 degrees to the right in relation to the patient face, created a non-polarized image with perspective distortion leading to the clinical misjudgment.

\section{Technical considerations relevant to plastic surgery}

Selecting a camera for use in plastic surgery telemedicine requires consideration of various capabilities and features. Consideration of key optical parameters such as color bit-depth, white balance, focus, and macro is important. As mentioned above, image quality cannot be low, however it cannot also be impractically high, requiring lengthy downloading time and lengthy transfer time. This can be especially problematic when transferring multiple high-pixel files from "the field" to the specialist. Therefore, excellent compression to reduce image file size and retain relevant details is a valuable and desirable feature (Patricoski C et al., 2010). There are two general types of image compression: "lossy" and "lossless." Lossless compression guarantees that the process of compressing and decompressing an image will 
not alter the image in any way. However, the rate of image compression is relatively low, typically 2:1. Much better rates of compression can be achieved by using Lossy compression. Lossy compression systems discard some information from the image, usually by removing small differences in pixel color that, ideally, are inconsequential (e.g., plastic surgery documentation frequently uses Joint Photographic Expert Group, JPEG)(Jones SM et al, 2004). In plastic surgery applications it is important to test the image compressing ratios and determine the maximum compression of the image for speedy transfer that will retain the minimum image quality for accurate clinical decision-making from the recipient (Patricoski C et al, 2009).

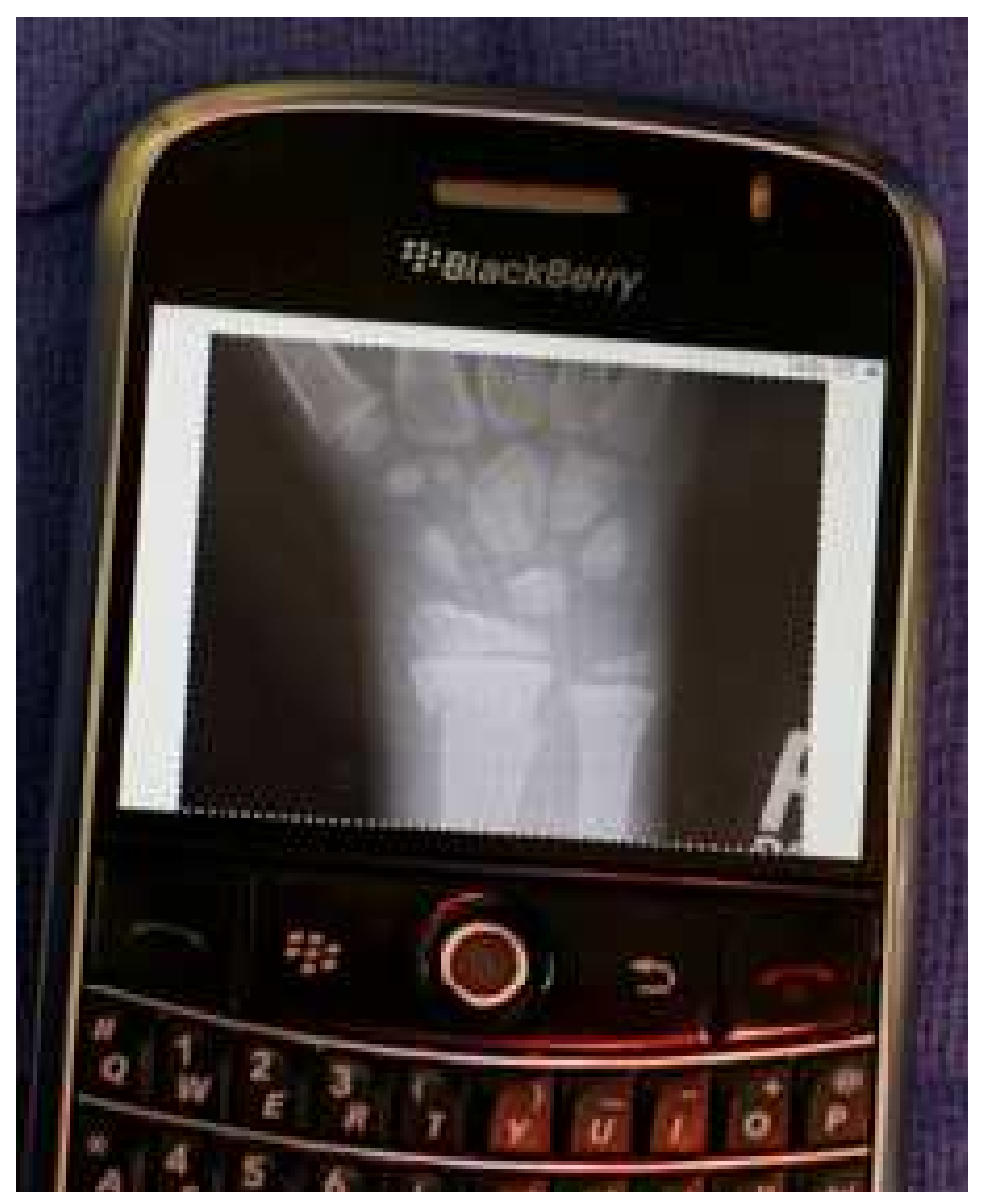

Fig. 3. Telemedicine in action: quick communication between the primary care physician office and surgical specialist streamlines decisions regarding how quickly the patient should be seen. In this case simple transmission of patient's x-ray facilitated his care: the patient who fell on outstretched hand the evening before was asked to come see a surgeon for reduction of his radial fracture on the same day he saw his primary care physician, and actually was scheduled for surgery prior to his arriving to the Plastic Surgery Clinic. 
In the case of videoconferencing, with dynamic image transfer there can be network bandwidth limitations from "the field" or at the specialist's location. Frequently, teleconferencing with clinics in remote or international locations which only have access to basic network systems necessitates a bandwidth quality that may be a communication obstacle. In this case, the teleconference becomes limited to the slowest bandwidth in the conversation.

Overall, however, cutting-edge technologies supporting telemedicine are used in wound care and plastic surgery. For example, a camera coupled to a hand held computer for data registration and encrypting that meets regulatory medical records requirements. Additionally, there are systems which interface with existing health care systems that facilitate care delivery (Fig. 3).

It is imperative that the access to and transfer of medical information by telemedicine modalities meet regulatory requirements regarding patient privacy (e.g., HIPAA in the United States or ISDN security in the United Kingdom). By equipping healthcare workers with mobile handheld computers which offer the security required for sensitive healthcare data transmission (e.g., ES400, Enterprise Digital Assistant (EDA) by Motorola, US), physicians and others healthcare professionals will be able to exchange data, communicate, and collaborate rapidly. The Silhouette Mobile by Aranz Medical in New Zealand is a portable, hand-held computer device with an integrated high-resolution camera designed specifically for the wound care and plastic surgery professional. This camera has embedded laser lighting for automated image calibration. Silhouette exports data in standard formats (running Windows Mobile 5 operating system) and is designed to support HIPAA compliance. These mobile devices will ultimately increase access and quality of care.

\section{Patients' perceptions}

The literature and our own observations indicate that telemedicine is well received by patients. Fears that patients will reject "anonymous" electronic communication between the patient and physician, or physician and specialist, appear unsubstantiated.

During our studies of implementing telemedicine as a decision aid for patients with chronic wounds we also evaluated the impact of telemedicine on the patient. This was measured by assessing the duration of the face-to-face consultation after or without intermediate telemedicine consult, the patient's satisfaction with care decisions, and a decisional conflict scale. The average face-to-face consultation was decreased from $50 \pm 12$ minutes to $35 \pm 6$ ( $p$ $<0.01$ ) minutes for patients who had received a telemedicine consult prior to the direct consultation. Moreover, patient satisfaction with care decisions was dramatically increased (93\% vs. $47 \%$ ) when patients were involved in a preliminary telemedicine consult. Patients experiencing the added initial telemedicine consult reported improvement in both the understanding of their care and in their perceived involvement in shared decision making regarding their care. Finally, the decision conflict scale, which measures uncertainty over wound care management plans, was reduced from $35 \pm 4.26$ to $14 \pm 1.73(\mathrm{p}<0.001)$ in patients subjected to telemedicine decision aid. This shows that there is added confidence in the wound care management plan when a telemedicine consult is included in the treatment plan (Dobke MK et al., 2008).

Telemedicine consultations provide accurate assessment for the treatment of problem wounds. They are well received by patients and have the potential to expedite and streamline care for patients with chronic wounds. The addition of telemedicine as an initial, 
intermediate communication step with a patient seems to educate the patient and ultimately enhances a bond with a specialist-consultant.

The following two examples illustrate positive outcomes for patients who experienced telemedicine in a long-term and interactive manner. Fourteen patients with traumatic brain injury underwent a series of 60 Internet-based cognition rehabilitation sessions to foster their acquisition of compensatory strategies to diminish memory loss and other cognitive impairments as well as to help increase quality of life (Berguist TF et al, 2010). Traditionally this rehabilitation is long, face to face, intensive, and patients are frequently confronted with the usual barriers to access rehab/medical centers (transportation, cost, etc.) The teleconferencing patient participants were subsequently surveyed regarding their satisfaction and comfort with the experience as well as their willingness to receive this mode of care again. The study showed that the patients were not only willing to participate in cognitive rehabilitation treatment over the internet, but that they were satisfied with the treatment and would be willing to receive care the same way again. Such positive outcomes for long-term rehabilitation lends support for new endeavors in plastic surgery to provide long-term rehabilitation to wound-care and reconstructive surgery patients, such as speech therapy for repaired cleft palate patients.

Telepractice is another application of telecommunications technology to deliver professional services at a distance by linking a clinician to client (American Speech-Language Hearing Association, 2005). In an analysis of telepractice as a tool for training parents as agents of intervention for children with early autism, satisfaction was surveyed (Baharav, E \& Reiser C, 2010). Parents were found to be comfortable with the telemedicine technology, and stated that it was essential in the treatment of their child. They stated a preference for continuing with the use of telepractice to deliver therapy to their child and that they would recommend it to others. This is an example of how, in the future, physicians or health care providers would be able to provide support, education and fast answers to questions of family members who are caring for loved ones with a disease or condition that requires intensive health care education.

Finally, in separate studies within transcultural and translingusitical environments (e.g., a Cleft Lip and Palate Program in Mexico coordinated from the U.S.), indicate very good reception of telemedicine as a modality of contact between a consultant and childrens' parents regardless of linguistical, cultural or economic differences (Brigden M et al, 2008; Kipps K. et al, 2010).

\section{Physicians perceptions}

A survey of physicians who are clients of telemedicine services indicates overall good satisfaction rates and revealed that telemedicine could result in an earlier initiation of treatment than conventional delivery of care (Bridgen $\mathrm{M}$ et al, 2008). Additional studies indicate that experience with telemedicine results in more positive attitudes towards this technology and more opportunities for use in their practice. Concerns that a telemedicine consultant may take over patient management and negatively impact the primary care health care practitioner practice, as espoused, by those questioning the suitability of the teleconsultations, are unfounded. Potential problems with continuity of care and uncertainty about the follow up process were not experienced. (Brigden et al, 2008; Dobke et al, 2006; Dobke et al, 2008; Hanson D et al, 2009). Observations of members of a university center and "field" plastic surgery team did not reveal concerns that there might be generational 
differences in acceptance of telemedicine technology, particularly in the case of plastic surgery health care providers (Loera JA, 2008). Members of plastic surgery teams indicated that the actual primary benefit of telemedicine availability is an improvement in the ability to consult others. This benefit ranges from immediate intra-operative consultations, to supporting isolated clinicians, enhancing education, and providing peer review.

There are some potential problems that have been raised by physicians when surveyed. Some general issues, not necessarily pertaining to plastic surgery, that have been raised include: problems with continuity of care, a need for improving patient comfort level with the telemedicine technologies, and absence of a complete physical examination (Bridgen $\mathrm{M}$ et al, 2008). Potential solutions for improving continuity of care would be avoiding referral for services that could be delivered locally, communicating a clear follow-up plan and having the specialist follow-up directly with the local family physician. To improve patient comfort with the technology a pre-orientation for new patients was suggested as well as the provision, for language difficulties, of an available and reliable translation service and technical coordinator during the consultation. As for improving the unavoidable lack of a complete physical exam, it was suggested that the local referring physician, just as is frequently practiced by community or rural emergency departments, be present to the consultation so they could provide an extra pair of hands for an exam directed by the specialist during the teleconference.

Ultimately, the success of telemedicine for patient diagnosis and treatment depends on the support and willingness of physicians and other healthcare providers (Hanson D et al., 2009). This motivation often depends on physicians experience and attitudes with prior telemedicine experiences even it is just a single prior exposure. As described above, experience with telemedicine increases a physician's predisposition to use telemedicine in their practice and even augments the breadth of implementation in different facets of his or her practice. Other ways to enhance physician acceptance is to have support from a sponsoring organization, provide structural legitimacy and a cohesive network (May et al, 2003).

\section{Video conferencing as an operational, research and educational tool}

The effectiveness of two way video conferencing for transmitting live video and audio of craniofacial consultations and reconstructive operations was evaluated for its potential to facilitate intra-operative consultations and enhance quality of instruction for students (with live surgery in a remote location). Prior research demonstrated that students who participated via videoconferencing remotely asked four times as many questions of the faculty, and vice versa faculty to students, than occurred when students were physically present in the operating room. Feedback from the study showed that students gained more from the telemedicine experience than from being physically in the operating room as measured by several objective and subjective criteria (McIntyre T et al., 2008).

A craniofacial operation was specifically selected for use as a telemedicine case report due its complexity, frequent need for intraoperative discussion, reliance on the input from multidisciplinary consultants, and, in the studied case, the international approach. Unlike telementoring projects which are hierarchical in nature, where one party is the teacher and the other party is the student, this project was more of a collaboration. There is a telemedicine unit in the children's hospital operating room at the Hospital Infantil de las Californias in Tijuana, Mexico that authors and their associates currently use to record and 
watch operations while they occur in real time. This is a specifically ordered unit for telemedicine purposes from Lifesize Communications, a division of Logitech. The computer is connected to a high definition camera and microphone on top of a videocamera crane. This allows for visualization of operations while they are occurring, both in high definition and with a bird's eye view.

The operating room teleconference unit has been a popular education tool for University of California San Diego, School of Medicine students. Students are able to watch operations in optimal video and sound quality from a classroom in La Jolla, California. Frequently a nonoperating member of the team may speak to students focusing on educational aspects of the surgery without taking time and focus away from the operating surgeon. Students are able to talk and ask questions of the surgeons directly, albeit briefly, while they operate. This has been a convenient and uniquely accessible venue for exposing students to pediatric plastic surgery, international health and volunteer work for underserved populations.

Telementoring may also serve as a sustainable way to bring mentors and trainees together in regions of the world with a shortage of certain specialists, educators, mentors, public facilitators and organizers of care (Fig. 4).

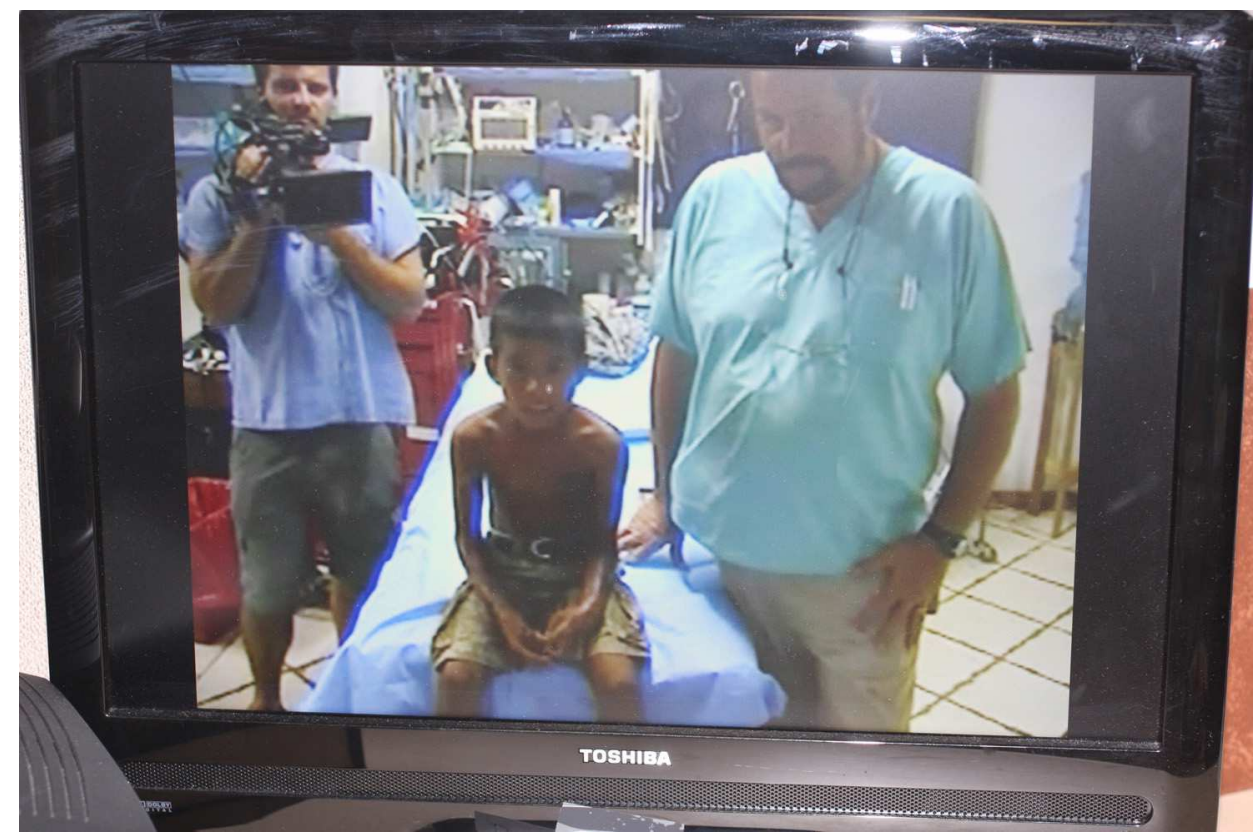

Fig. 4. Teleconferencing with the Peten (Guatemala) jungle "Plastic Surgery Clinic". This is a patient ( 9 year old boy) after surgical release of severe postburn axillary and antecubital fossa contractures. The patient is being guided by non-medical staff to demonstrate the range of motion of his upper extremities. A new physical therapy regime for selfimplementation is being discussed. The cameraman in the background is from Guatemalan TV making a film about telemedicine for public education.

In one case study, a 7-year-old male patient in Tijuana, Mexico received microvascular reconstructive surgery to treat hemifacial microsomia (Gosman AA, 2009). The standard of 
care for hemifacial microsomia, a complex congenital problem, is a multi-disciplinary team. A team of volunteer specialists from Mexico and the United States participated in this rare and technical operation. Additionally, during part of the procedure, a teleconference set up between the operating room at the Hospital Angeles in Tijuana, Mexico and a group of medical students, residents and plastic surgery specialists at the University of California, San Diego. In fact, teleconferencing was evaluated, for its ability to extend the multidisciplinary team beyond the walls of the operating room.

A low-tech videoconference system was set up using the free and universally available Skype software, which is both encrypted and compliant with Health Insurance Portability and Accountability Act regulations. The study evaluated the effectiveness of the two-way tele-communication for potential ad hoc consultations during the operation on an as needed basis. It was found that telemedicine would contribute to a multidisciplinary approach, and that it would in fact facilitate an improvement to follow-up with postsurgical patients from a distant location. After volunteer surgeons return to their respective hometowns, they along with other members of the multi-disciplinary team would be able to track the recovery of their patients remotely.

\section{Assessing outcomes and providing long-term care in plastic surgery}

Another area of ongoing research in telemedicine is to evaluate its use as a tool to assess outcomes in plastic surgery, such as in cleft and palate surgery (Fig. 5). In particular, we are studying it as a means of providing effective speech therapy for cleft palate patients. This exemplifies the potential and rapidly growing application of telemedicine in reconstructive surgery as well as its increasing role as a clinical practice, research and plastic surgery education enhancing tool.

Our current, across-the-border telemedicine projects, in transcultural environments, (San Diego to Tijuana, Mexico and San Diego to a medically unsupervised location in the jungle of Peten, Guatemala), include a collaboration with the Cleft lip and Palate Clinic in Tijuana, Mexico based at the Hospital Infantil de las Californias (to which a reference was already made above). Here we are performing an evaluation of whether speech therapy can be provided over a teleconference unit. Bilingual speech pathologists evaluate patient speech in real-time from San Diego, California. The child sits in front of a camera and video screen and follows the directions of the speech pathologist. Frequently, the child's parent or guardian will have to help manage the encounter and provide support to certain speech exercises such as assisting in holding closed the child's nose to prevent nasal emission. The equipment used for this collaboration includes two teleconference systems, one located in a physician's office in San Diego, California and the other is in the patient exam room of the Hospital Infantil in Tijuana, Mexico. The San Diego office utilizes a Polycom Viewstation teleconference system with a variable zoom $(5.4-64.8 \mathrm{~mm})$ camera and the exam room in Tijuana is visualized using a TV screen. The exam room in Tijuana uses a desktop computer with videocamera and monitor for its teleconference, using a program called Viewpoint HD. Preliminary experience demonstrates that transmission of acoustic data may be equally challenging as the transmission of visual images.

This study is valuable because it may provide experience in telemedicine coupling vision and voice data. It may show that it is not only possible to provide speech therapy but also assess outcomes without being face-to-face, which could increase access to many patients in 
Mexico who are in great need for speech therapy. This would conceivably alleviate a large unmet medical need to an underserved population that does not currently have access to speech therapy. In fact, there does not even exist a speech therapy profession in Mexico. Additionally, this could potentially be a model system for other international telemedicine therapy programs that would be able to provide continuous care treatment beyond the more typical use of telemedicine which has a focus on individual one-time case based interactions. As described earlier, local studies in telepractice have proven positive results integrating long-term treatment plans to parents of children with autism and to brain injury patients undergoing cognitive rehabilitation.

It appears that the delivery of healthcare through telemedicine in the field of plastic surgery to medically underserved patient populations, or even unsupervised settings is safe and meets demands of rural or wilderness settings. Plastic surgery surgical intervention outcomes did not seem to be impacted negatively by the telemedicine and remote management of certain components of the overall care. Patient participation in our telemedicine programs was completely voluntary, and yielded a high satisfaction rate reaffirming experience of other investigators (Nesbitt TS et al., 2000). Also, experience with

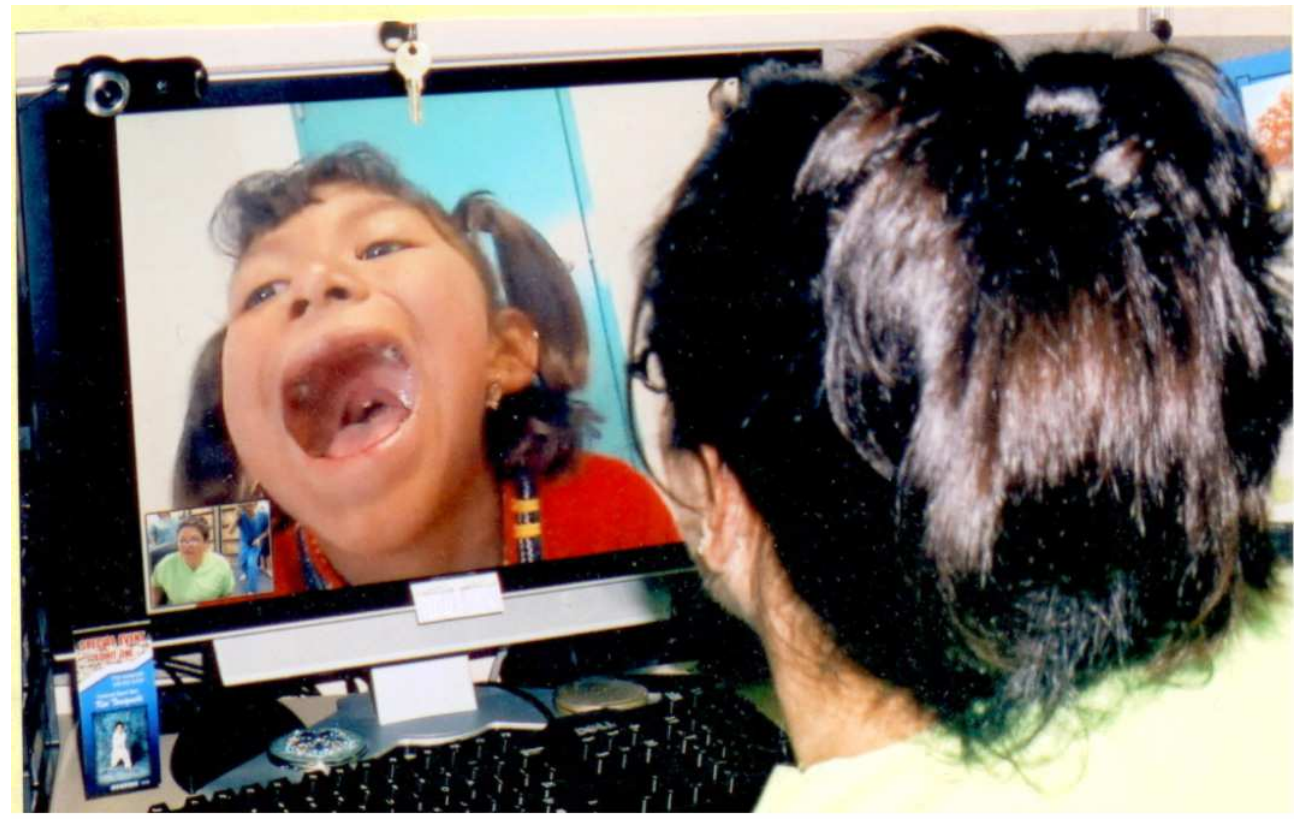

Fig. 5. Video-conferencing with a medically unsupervised remote Plastic Surgery Clinic in the jungle of Peten (Guatemala). The patient demonstrates to the nurse located in the Clinic in the US how well her repaired cleft palate healed. It is possible to provide basic speech assessment during video conferences as depicted in the photograph. Noteworthy is this patient's (and other children) eagerness to pose to the camera. This "Hollywood-Effect" observed when patient poses to the camera vanishes frequently, during a face-to-face encounter with a health care provider armored with a tongue blade: children frequently do not cooperate. 
telemedicine consults indicate that the transcultural delivery and the presence of interpreters does not reduce the patient and or families satisfaction rates (Kipps K. et al, 2010)

Many telemedicine projects were implemented in remote areas where, by necessity, technical arrangements were limited by availability of Internet and technical equipment. However, wherever possible, the video teleconsultation should consist of a secured Internet Web-based application, a personal or even hand-held computer, a broadband Internet connection and a digital camera. There is an increasing body of evidence that video consultation has added value to the quality of care in plastic surgery and other specialties (Visser et al, 2010).

\section{Future of telemedicine in plastic surgery}

As technologies supporting telemedicine are developed and perfected they will help to advance plastic surgery into the digital era. Telemedicine will certainly help in globalization of plastic surgery delivery. This technology will be instrumental in addressing basic needs in medically underserved areas and the most complex surgical needs by enabling sophisticated expert consultations. With both technological advancements and growing physician exposure, telemedicine will continue to thrive in increasing applications.

Ultimately, telemedicine will be essential for the development of remotely steered or assisted procedures, both robotic and traditional, performed by a human health care professional. This too will be utilized to improve access to specialists in plastic surgery in remote national and possibly international locations.

Finally, the development of home telemedicine programs for direct communication between a plastic surgeon and a patient at home may facilitate an improved quality of care. This could occur, for example, by means of as little as a cellular phone with a camera. This will hopefully enhance postoperative follow-up by improving time spent between specialist and patient.

\section{References}

American Speech-Language Hearing Association. Speech-language pathologists providing clinical service via telepractice: Position Statement. Available at http://www.asha.org/docs/html/PS2005-00029.html. Accessed August 23, 2010.

Association for the Advancement of Wound Care Statement on Comprehensive Multidisciplinary Wound Care. Available at: http://www.aawconline.org/pdf/AAWCMulti-disciplinarystatement.pdf. Accessed May 2, 2007.

Baharav E \& Reiser C. Using telepractice in parent training in early autism. Telemedicine and e-Health, 16(6) 1 - 5, 2010.

Berguist TF, Thompson K, Gehl C, \& Muno Pineda J. Satisfaction ratings after receiving Internet- based cognitive rehabilitation in persons with memory impairments after severe acquired brain injury. Telemedicine and e-Health, 16(4) 1- 7, 2010.

Bridgen M, Minty A, Pilatzke S, Vidova LD, Sherrington L, \& McPhail K. A survey of recipient client physician satisfaction with teleoncology services origionating from 
Thunder Bay Regional Health Sciences Centre. Telemedicine \& e-Health, 14(3), 250 254, 2008.

Dobke M, Renkielska A, De Neve J, Chao J, \& Bhavsar D. Telemedicine for problematic wound management: Enhancing communication between long-term care, skilled nursing, and home caregivers and a surgical wound specialist. WOUNDS: A Compendium of Clinical Research and Practice, 18(9), 256-261, 2006

Dobke MK, Bhavsar D, Gosman AA, De Neve J, \& De Neve B. Streamlining the management of patients with problematic wounds: Must a multidisciplinary team formulate all patient management plans? WOUNDS: A Compendium of Clinical Research and Practice, 19(12), 340-344, 2007

Dobke MK, Bhavsar D, Gosman A, De Neve J, \& De Neve B. Pilot trial of telemedicine as a decision aid for patients with chronic wounds. Telemedicine and E-Health, 14(3), 245249, 2008

Dobke MK \& Gosman A. Applications of telemedicine in aesthetic surgery. Wound Management, 6(2), 52, 2009

Gosman AA, Fischer CA, Agha Z, Sigler A, Chao J, \& Dobke MK. Telemedicine and surgical education across borders: A case report. Journal of Surgical Education, 66(2), 102-105, 2009

Hanson D, Calhoun J, \& Smith D. Changes in provider attitudes toward telemedicine. Telemedicine \& e-Health, 15(1), 39-44, 2009

Jones SM, Banwell PE, \& Shakespeare PG. Telemedicine in wound healing. International Wound Journal, 1(4), 225-230, 2004

Kipps K, Sigler A, Bailey J, Dobke M, \& Gosman A. Telemedicine and international craniofacial reconstruction: patient perspectives (abstract). California Society of Plastic Surgeons, 60th Annual Meeting, Rancho Palos Verdes, CA, May 27-31, 2010

Loera JA. Generational differences in acceptance of technology. Telemedicine $\mathcal{E}$ e-Health, 14(10), 1087 - 1090, 2008

May C, Harrison R, MacFarlane A, Williams T, Mair F, \& Wallace P. Why do telemedicine systems fail to normalize as stable models of service delivery? J Telemed Telecare, 9(suppl 1):25-26, 2003

McIntyre T, Monahan T, Villegas L, Doyle J, \& Jones DB. Teleconferencing surgery enhances effective communication and enriches medical education. Surg Laparosc Endosc Percutan Tech, 18, 45-48, 2008.

Meyer BC \& Friedman LS. (2010). Telemedicine - closing in on distance medicine. San Diego Physician, 97(7), 36 - 39, 2010

Nesbitt TS, Hilty DM, \& Siefkin A. Development of a telemedicine program. Western J Med, 173(3), 169 - 174, 2000

Patricoski C, Ferguson AS, Brudzinski J, \& Spargo G. (2010). Selecting the right digital camera for telemedicine - choice for 2009, Telemedicine and e-Health, 16(2), 1 - 8, 2010

Tadros A, Murdoch R, \& Stevenson JH. (2009). Digital image referral for suspected skin malignancy - a pilot study of 300 patients. J Plast Reconstr Aesth Surg, 62(8), 1048 1053, 2009 
Terry M, Halstead LS, O'Hare P, Gaskill C, Ho PS, Obecny J, James C, \& Lauderdale ME. Feasibility study of home care wound management using telemedicine. Adv Skin Wound Care, 22(8), 358 - 364, 2009

Visser J, Bloo JK, Grobbe FA, \& Vollenbroek-Hutten MMR. Video teleconsultation service: who is needed to do what, to get it implemented in daily care? Telemedicine $\mathcal{E} e$ Health, 16(4), 439 - 445, 2010 


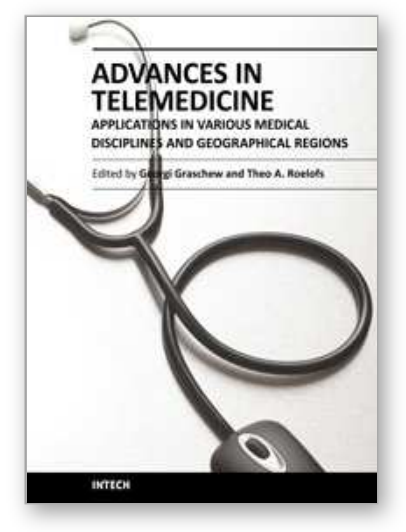

\author{
Advances in Telemedicine: Applications in Various Medical \\ Disciplines and Geographical Regions \\ Edited by Prof. Georgi Graschew
}

ISBN 978-953-307-161-9

Hard cover, 296 pages

Publisher InTech

Published online 22, March, 2011

Published in print edition March, 2011

Innovative developments in information and communication technologies (ICT) irrevocably change our lives and enable new possibilities for society. Telemedicine, which can be defined as novel ICT-enabled medical services that help to overcome classical barriers in space and time, definitely profits from this trend. Through Telemedicine patients can access medical expertise that may not be available at the patient's site.

Telemedicine services can range from simply sending a fax message to a colleague to the use of broadband networks with multimodal video- and data streaming for second opinioning as well as medical telepresence. Telemedicine is more and more evolving into a multidisciplinary approach. This book project "Advances in Telemedicine" has been conceived to reflect this broad view and therefore has been split into two volumes, each covering specific themes: Volume 1: Technologies, Enabling Factors and Scenarios; Volume 2: Applications in Various Medical Disciplines and Geographical Regions. The current Volume 2 is structured into the following thematic sections: Cardiovascular Applications; Applications for Diabetes, Pregnancy and Prenatal Medicine; Further Selected Medical Applications; Regional Applications.

\title{
How to reference
}

In order to correctly reference this scholarly work, feel free to copy and paste the following:

Marek Dobke and Elizabeth Whitehead (2011). Telemedicine as an Aid to Clinical Practice, Research and Education in Plastic Surgery, Advances in Telemedicine: Applications in Various Medical Disciplines and Geographical Regions, Prof. Georgi Graschew (Ed.), ISBN: 978-953-307-161-9, InTech, Available from: http://www.intechopen.com/books/advances-in-telemedicine-applications-in-various-medical-disciplines-andgeographical-regions/telemedicine-as-an-aid-to-clinical-practice-research-and-education-in-plastic-surgery

\section{INTECH}

open science | open minds

\author{
InTech Europe \\ University Campus STeP Ri \\ Slavka Krautzeka 83/A \\ 51000 Rijeka, Croatia \\ Phone: +385 (51) 770447 \\ Fax: +385 (51) 686166 \\ www.intechopen.com
}

\author{
InTech China \\ Unit 405, Office Block, Hotel Equatorial Shanghai \\ No.65, Yan An Road (West), Shanghai, 200040, China \\ 中国上海市延安西路65号上海国际贵都大饭店办公楼 405 单元 \\ Phone: +86-21-62489820 \\ Fax: +86-21-62489821
}


(C) 2011 The Author(s). Licensee IntechOpen. This chapter is distributed under the terms of the Creative Commons Attribution-NonCommercialShareAlike-3.0 License, which permits use, distribution and reproduction for non-commercial purposes, provided the original is properly cited and derivative works building on this content are distributed under the same license. 